\title{
Spatial access to in-patient health care in northern rural India
}

\author{
Vikram Ranga ${ }^{1}$, Pradeep Panda ${ }^{1}$ \\ ${ }^{1}$ Micro Insurance Academy, The Sarvjan Unnati Bodhini (SUB) Charitable Trust, New Delhi, India
}

\begin{abstract}
Access to health care in rural areas is a major concern for local populations as well as for policy makers in developing countries. This paper examines spatial access to in-patient health care in northern rural India. In order to measure spatial access, impedance-based competition using the Three-Step floating Catchment Area (3SFCA) method, a modification of the simple gravity model, was used. 3SFCA was chosen for the study of the districts of Pratapgarh and Kanpur Dehat in the Uttar Pradesh state and Vaishali in the Bihar state, two of India's poorest states. This approach is based on discrete distance decay and also considers more parameters than other available methods, hence is believed to be a robust methodology. It was found that Vaishali district has the highest spatial access to in-patient health care followed by Pratapgarh and Kanpur Dehat. There is serious lack of health care, in Pratapgarh and Kanpur Dehat with $40 \%$ and $90 \%$ of the villages having shortage of in-patient care facilities in these respective districts. The most important factor affecting spatial access was found to be the distance to the nearest major urban agglomeration.
\end{abstract}

Keywords: 3SFCA, spatial access, in-patient health care, India.

\section{Introduction}

Health care access is a major concern for policy makers globally. It represents the degree of fit between the clients and the health care system (Penchansky and Thomas, 1981; McIntyre et al., 2009) and is a multistage and multidimensional concept, where the stages are defined as potential and realised (Joseph and Phillips, 1984; Guagliardo, 2004), while the dimensions are availability, accessibility, affordability, acceptability and accommodation (Penchansky and Thomas, 1981). Geographical information system (GIS) research emphasises the spatial dimension i.e. accessibility (McLafferty, 2003), while the rest of the dimensions may be seen as non-spatial. Potential accessibility assumes that all members of a population (i.e. a centroid/population weighted centroid) are potential users of the medical facilities present within a defined distance or driving time (a catchment area). On the other hand, realised accessibility is the actual use of the health care provider by the members of a population defined in this way. Potential accessibility is generally used to identify areas short of health care providers and therefore a useful measure for policy makers for allocating health services. Much GIS research in the health care field focuses on method-

Corresponding author:

Vikram Ranga

Micro Insurance Academy

The Sarvjan Unnati Bodhini (SUB) Charitable Trust

52-B, Okhla Industrial Estate, Phase-III, New Delhi, India

Tel. +91 11 4379-9100; Fax +91 11 4379-9117

E-mail: vikramranga@mia.org.in ological development making it more robust for assessing potential spatial access (Higgs, 2004). These methods are therefore frequently changing or modified (Radke and Mu, 2000; Guagliardo, 2004; Luo, 2004; Luo and Qi, 2009; Wan et al., 2012a).

The simplest way to determine accessibility is to assess the ratio of health care providers to population within an administrative boundary. Despite simple and intuitive, this approach fails to account for crossboundary travel by patients seeking care, a limitation resulting in the floating area catchment method (Luo, 2004). The gravity model is considered the best for evaluating spatial accessibility (Guagliardo, 2004) but not intuitive when it comes to interpretation (Luo and Qi, 2009). It also has problems with the continuous distance-decay function in absence of detailed traffic information. Radke and $\mathrm{Mu}$ (2000) proposed a twostep catchment area, which was eventually modified into a two-step floating catchment area (2SFCA) method by Luo (2004) and Wang and Luo (2005), the latter itself being a modification of the simple gravity model (Wan et al., 2012a) to account for its limitations. Luo and Qi (2009) modified the 2SFCA and called it the enhanced 2SFCA (E2SFCA). Recently, (Wan et al., 2012a) proposed a further modification to E2SFCA, namely the three-step floating catchment area (3SFCA) method. This method added impedancebased competition to the E2SFCA approach, e.g. if two health care providers are present within a catchment zone, the chance of choosing one of them decreases due to the possibility of turning to the other. The major difference between the 3SFCA and the gravity model is that latter uses continuous distance 
decay, while the former uses discrete distance decay, considered as more logical in practical situations (Luo and Qi, 2009; Wan et al., 2012a). Finally, there is the kernel density estimation (KDE) approach (Silverman, 1986; Guagliardo, 2004; Spencer and Angeles, 2007) but in a head-to-head comparison with the 2SFCA method using the same area, the latter was reported to be superior (Yang et al., 2006).

Economically better-off regions usually have better access to health care, i.e. rich countries have better access than poor countries (Peters et al., 2008) and urban populations have better access than rural ones (Krishna and Ananthpur, 2013). In India, despite economic growth and reforms, child health problems and maternal mortality remain unacceptably high (Baru et al., 2010) indicating a failing health system, perhaps India's greatest, current predicament (Horton and Das, 2011). This was identified soon after independence and following the Bhore committee recommendation (Qadeer, 2011) to aim at equal health care to all; the rural health care infrastructure was designed as a three-tier system: sub-centres (SC), primary health centres (PHC) and community health centres $(\mathrm{CHC})$, wich was meant for populations of 5,000, 30,000 and 120,000, respectively (Ministry of Health and Family Welfare, 2012). Although these facilities were meant to provide high-quality services in public health, they failed to do so due to lack of health care professionals; either this section of the staff could not be recruited or they did not turn up for duty after being appointed (Banerjee et al., 2004; Sengupta and Prasad, 2011; Ministry of Health and Family Welfare, 2012). De Costa and Diwan (2007) observed, in the Indian Madhya Pradesh state, that the problem has more to do with allocation and quality than manpower. However, after the National Rural Health Mission (NRHM) was launched in 2005 to find a solution to health care accessibility in the rural areas, the latest survey (March 2011) still shows shortfall of health care professionals in all three aforementioned tiers (Ministry of Health and Family Welfare, 2012). Deshpande et al. (2004) and De Costa and Diwan (2004) attempted to figure out the general practices in the rural areas based on geographic concepts but a modern, robust quantification of spatial access in rural settings has yet to be tried.

With this in mind, we initiated a study aiming at answering the following questions:

(i) What is the spatial access with reference to inpatient care in rural settings?

(ii) How does spatial access vary among different rural areas?

(iii) Which parameters control the variations in the spatial access among different, rural areas?
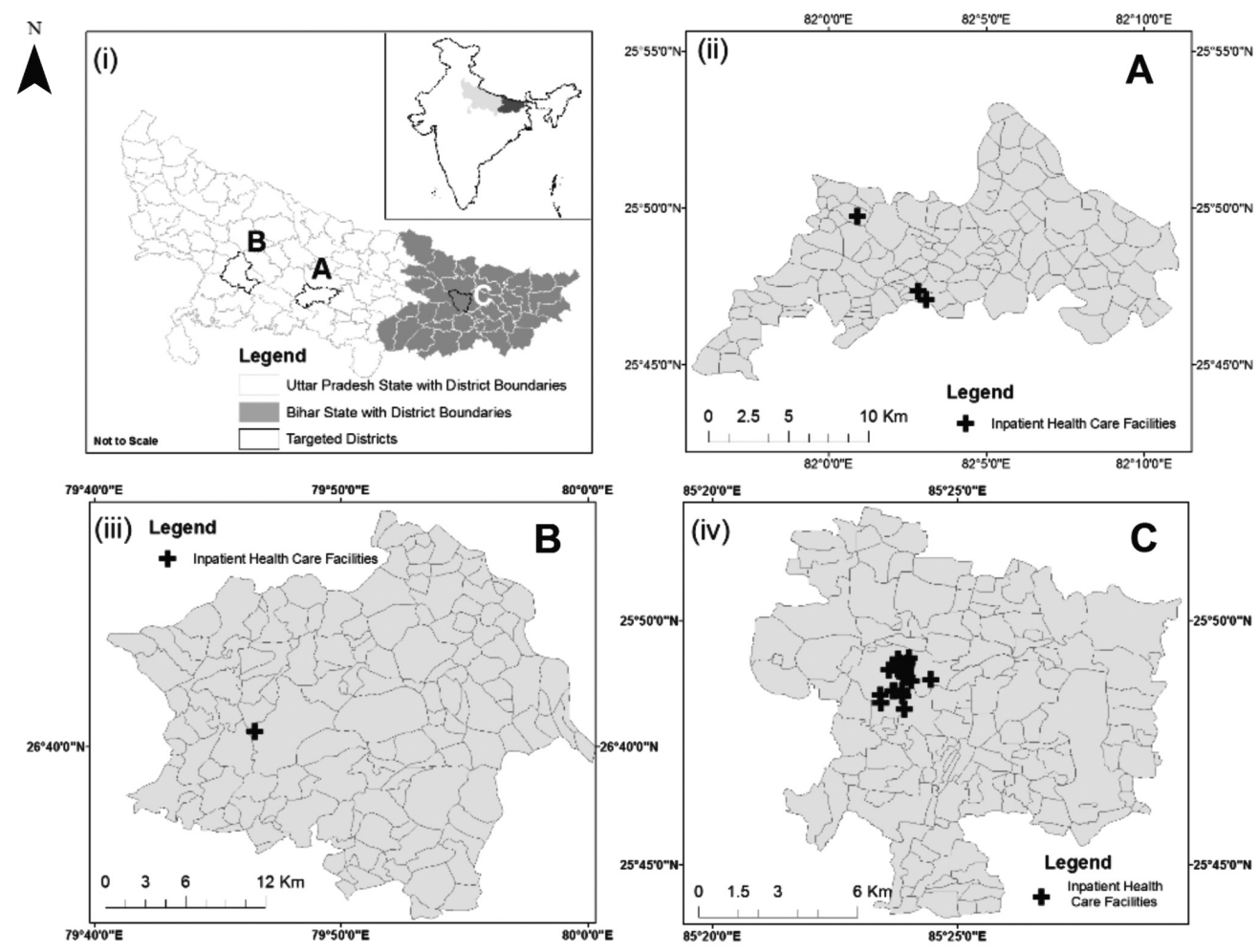

Fig. 1. Location of the states of Uttar Pradesh and Bihar (i) with inset of India and the three study sites Pratapgarh (ii), Kanpur Dehat (iii) and Vaishali (iv). 


\section{Materials and methods}

This work is a part of a 5-years, community-based health insurance initiative undertaken by Micro Insurance Academy (New Delhi, India) in collaboration with Erasmus University (Rotterdam, The Netherlands) and University of Cologne (Cologne, Germany). The selection criteria and detailed protocol for this study has been published elsewhere (Doyle et al., 2011; Dixit and Panda, 2013). Therefore, only the particular information used in this study is explained here.

\section{Study area}

The study took place in three blocks (districts), i.e. Shivgarh (Pratapgarh) and Rasoolabad (Kanpur Dehat) in the state of Uttar Pradesh and Mahua (Vaishali) in Bihar state (Fig. 1) covering areas of 166, 551 and $134 \mathrm{~km}^{2}$, respectively. The study areas did not represent the blocks completely but covered most of these areas. All three sites are poor, rural areas on the Ganges River plains primarily dependent on agriculture. They are located outside major urban centres, namely Allahabad, Kanpur and Patna at approximate road distances of $71 \mathrm{~km}, 73 \mathrm{~km}$ and $43 \mathrm{~km}$, respectively. The climate is humid semi-arid and designated as Cwa in the Köppen-Geiger classification (Peel et al., 2007).

Table 1. Health care providers covered in the supply-side survey.

\section{Datasets}

For health care accessibility studies, both demand side (the need of health care) and supply side (hospitals, health centres, etc.) needs to be covered. For both sides, detailed questionnaires were used for data retrieval: (i) quantitative data from a supply-side health care provider survey and a demand-side household survey; and (ii) spatial supply-side location data, collected with a hand-held Garmin etrex vista global positioning system (GPS) instrument, and demandside spatial data at the village level extracted from census maps available at the Census of India administration (http://www.censusindia.gov.in/maps/maps_prod/ Map_Product_State.aspx). The supply side health care provider data were collected in May-June 2010 and covered 3,092 of all types of health care providers, but only in-patient care facilities were used (three in Pratapgarh, one in Kanpur Dehat and 30 in Vaishali) (Table 1). The demand-side household data ${ }^{1}$ (including 3,685 households) were collected in March-May 2010. The areas covered by the two surveys were not equal as the demand-side coverage are subsets of the supply-side coverage ${ }^{2}$ (for detailed household survey coverage see Doyle et al. (2011) and Dixit and Panda (2013). The revenue ${ }^{3}$ village was the best resolution as it could be extracted with exact population details from census records. The road network was digitised from a satellite image produced by a linear imaging self scanning sensor (LISS-IV; spatial resolution $=5.8$ m) on-board Resourcesat - 2 satellite (http://bhuvan.nrsc.gov.in). All questionnaires were entered into Excel spreadsheets and analysed using the R software (R Development Core Team, 2012).

\footnotetext{
${ }^{1}$ The randomised control trial (RCT) design was used for household survey sampling and covered only members affiliated with self-help groups, while the provider survey was based on census of all health care providers in the study areas.

${ }^{2}$ Normally, people go to doctors of their choice. However, these doctors might not live in their village but be situated at a significant distance away from the village. Therefore, a surrounding area based on key informant interveiws (KII) and focus group discussions (FGD) described in Doyle et al. (2011) was used to cover all health care providers where local people (covered in demand-side surveys) generally go.

${ }^{3}$ The revenue village is a separate administrative unit with village accounts and well-defined surveyed boundaries, often consisting of one or more hamlets rather than a single agglomeration of habitations (http://censusindia.gov.in/Data_Products/ Library/Indian_perceptive_link/Census_Terms_link/censusterms.html).
} 


\section{Methodology}

Spatial access

The spatial access was calculated using the 3SFCA method (Wan et al., 2012a). The key idea behind using accessibility is that access to health care providers is recognised as an important facilitator of the overall population health (Guagliardo, 2004), which helps to narrow down areas with shortages of health facilities thereby supporting decision-making. The 3SFCA method uses the distance decay function, which is calculated using the Gaussian distance decay $\left(f(d)=e^{-d^{2} / \beta}\right.$; for details see Kwan, 1998). Three discrete distance zone were defined where sub-zones 2,5 and $10 \mathrm{~km}^{4}$ were used as $d$, while the impedance $(\beta)$ was assigned the value of 100 . The geometric centroids of revenue villages were considered population centres. Since there is lack of roads connecting centroids to health facilities, a relation was assessed between the road network distances and the Euclidean distance. To this end, using the CHCs as reference, random points $(\mathrm{n}=$ 100) were selected from the supply-side and the Euclidean distance was calculated using the "rgeos" package (Bivand and Rundel, 2013) from the CHCs to the locations of the 100 random health care providers. However, out of the 100 points, only those situated along marked roads could be used as not all 100 random health care providers were found on the roads mapped. Subsequently, the dataset used with the Euclidean distance was again used to calculate actual road distances in Quantum GIS (QGIS Development Team, 2013) with the road network plug-in facility. Travel time, instead of distance, is generally used to define catchment boundaries (Wang, 2002; Wang and Luo, 2005; McGrail and Humphreys, 2009; Wan et al., 2012a,b), therefore, a relation was assessed between travel distance and the time taken to travel that distance. For this relation, demand side/household data (sample size $=3,685$ ) was used, which included questions on distance travelled against the time used for that distance. The 3SFCA method was implemented in three steps described below.

${ }^{4}$ The values 2, 5 and 10 represent discrete distance zones. Since we defined subzones, it is no more a continuous distance decay but a discrete one. Kwan (1998) advocated the formula used in this study. There is currently no consensus of using a fixed value of impedance. However, it has been suggested by Wan et al. (2012b) to use the spatial access ratio (SPAR) for the final output, which is invariable with respect to the choice of impedance.
Step 1

For population location $(i)$, we based the catchment area (the centroid of village polygon) on a $10 \mathrm{~km}$ radial zone dividing it into three sub-zones of 2, 5 and 10 $\mathrm{km}$ radii, respectively. The in-patient facilities were assigned Gaussian weights depending on which zone they belonged to, after which we used equation 1 to calculate the selection weight for each population centre and inpatient care combination as follows:

$$
G_{i j}=\frac{T_{i j}}{\sum_{k \in\left[D i s t(i, k)<d_{0}\right]} T_{i k}}
$$

(equation 1)

where $G_{i j}$ is the selection weight between location $i$ and in-patient care location $j$ and $\operatorname{Dist}(i, k)$ the Euclidean distance and $d_{0}$ is the catchment area, i.e. 10 $\mathrm{km}$. The $G_{i j}$ is, essentially, unique for each combination of locations $i$ and $j . T_{i j}$ is the Gaussian weight for $j$, calculated by using Gaussian distance decay formula $\left(f(d)=e^{-d^{2} / \beta}\right)$, divided by summation of all the possible Gaussian weights of in-patient care (Wan et al., 2012a). This step introduces competition among service providers in the case of availability of more than one service provider within the catchment area with population location $i$.

\section{Step 2}

Subsequently, we determined the catchment and sub-zone as in the first step for each in-patient care facility. We searched all population centres and calculated the supply-to-demand ratio $\left(R_{j}\right)$ for $j$ using equation 2:

$$
\begin{gathered}
R_{j}=\frac{S_{j}}{\sum_{r=1,2,3} \sum_{k \in D_{r}} G_{k j} P_{k} W_{r}}= \\
\frac{S_{j}}{\sum_{k \in D_{r}} G_{k j} P_{k} W_{1}+\sum_{k \in D_{r}} G_{k j} P_{k} W_{2}+\Sigma_{k \in D_{r}} G_{k j} P_{k} W_{3}}
\end{gathered}
$$

(equation 2)

where $S_{j}$ is the number of in-patient care (which would always be equal to 1 in this case), $W_{r}$ the Gaussian weight for the $r$ th sub-zone area, $G_{k j}$ the selection weight between $k$ and $j$ and $P_{k}$ the population of $k$.

\section{Step 3}

We computed the spatial access index (SPAI) by equation 3 as follows:

$$
\begin{gathered}
A_{i}^{F}=\sum_{r=1,2,3} \sum_{j \in D_{r}} G_{i j} R_{j} W_{r}= \\
\sum_{j \in D_{1}} G_{i j} R_{j} W_{1}+\sum_{j \in D_{2}} G_{i j} R_{j} W_{2}+\sum_{j \in D_{3}} G_{i j} R_{j} W_{3}
\end{gathered}
$$

(equation 3) 
where $R_{j}$ comes from equation 2 and $G_{i j}$ is the selection weight between $i$ and $j$ from equation 1 . $W_{r}$ is the Gaussian weight of the $r$ th sub-zone. The implementation of 3SFCA method was coded in $\mathrm{R}$ ( $\mathrm{R}$ Development Core Team, 2012).

The $A_{i}^{F}$ in equation 3 represents SPAI, which can be considered as the supply-to-demand ratio (Wang and Luo, 2005; Luo and Qi, 2009; Wan et al., 2012b). Wan et al. (2012b) proposed spatial access ratio (SPAR) for analysis and mapping purposes but recommended the use of SPAI for identifying areas with shortage of health care professionals. SPAR is the SPAI of administrative boundary divided by the global SPAI mean and since SPAR is a normalised value, it is less variable in response to the choice of impedance value, while SPAI varies significantly with such change. Wan et al. (2012a) proposed a complementary measure to the 3SFCA method, the adjusted spatial access (ASPA), to identify areas with shortage of health care professionals. ASPA is an extension of SPAR and based on supplier competition (Wan et al., 2012a). For a simpler and more intuitive interpretation, SPAI was used here to identify areas with shortage of health care professionals, while SPAR was used for mapping purposes (Fig. 5).

\section{The Kruskal-Wallis test}

SPAI varies hugely among study sites and is also dependent on the choice of impedance. In addition, SPAR is dependent on the mean of SPAI, which may also vary hugely; therefore, neither SPAI nor SPAR is useful in a statistical model aimed at establishing relations with explanatory variables. However, it was possible to analyse the differences among the three sites and obtain the same trend as with spatial access. Thus, the hypothesis test was carried out with a null hypothesis of the studied parameters having the same distribution in all the three locations. The studied socio-economic parameters based on the Indian census $(2001)^{5}$ were as follows: total population (TOT_POP), total literacy (TOT_LIT), total illiteracy (TOT_ILLT), total cultivators (TOT_CULT), total non-workers (TOT_NNW) and distance (N_DIST) to nearest urban agglomeration $\left(\mathrm{UA}^{6}\right)$. The revenue village was used as

\footnotetext{
${ }^{5}$ These data were collected when the project started in 2010, at that time no later census than that of 2001 was available.

${ }^{6} \mathrm{An}$ urban agglomeration (AU) is a continuous urban spread constituting a town and its adjoining outgrowths. An UA must consist of at least a statutory town and its population should not be less than 20,000 as per 2001 (Census of India, 2011).
}

the observation unit (as in spatial access). All the socio-economic parameters were extracted from the census data of 2001; while the distance to nearest village was calculated from village centroids to nearest UA defined according to Census of India, 2011. As a preliminary step, spatial autocorrelation was assessed using the "spdep" package (Bivand, 2013) for all parameters; the weighting was kept as row-standardised. Since the observations were neither normally, nor homoscedasticly distributed, a non-parametric method, i.e. the Kruskal-Wallis approach (Aczel and Sounderpandian, 2006), was used. Subsequently, a pair-wise Wilcoxon rank-sum post-hoc test was performed with Bonferroni's adjustment (Diez et al., 2013) to the significance level of $\alpha$ for individual differences. The Kruskal-Wallis method indicates significant values, even if only one group is significantly different, it does not tell where the difference lies, which shows up when the post-hoc test is applied.

\section{Results}

\section{Catchment selection}

Road distance versus Euclidean distance

As pointed out by Yao et al. (2013), the use of Euclidean distance might not be ideal as it might ignore many physical barriers; therefore, a relation was assessed between Euclidean distance and the shortest road network distance. A significant relation was found between shortest route distance using road network and the Euclidean distance between two points. As shown in Fig. 2, variability explained by the regression line is very good (mean regression $\mathrm{R}^{2}=$ 0.94). The least variability in the case of Pratapgarh can be attributed to fewer roads than in the other two sites. For Vaishali, as expected, the range was found to be the least attributed to the smallest area. Similarly, the higher range for Kanpur Dehat was accepted as attributed to the bigger area. The use of travel time instead of distance was explored, but the linear relationship between travel time and distance travelled does not explain variability (mean regression $\mathrm{R}^{2}=$ 0.54). The reason can be attributed to bias due to the popular habit of rounding up time to the closest hour (Fig. 3). Furthermore, duration in travelling the same distance varied, indicating different modes of transportation, while the heterogenic road conditions made it difficult to consider speed at all. Considering these local situations, Euclidean distance was used for the subsequent analyses. 


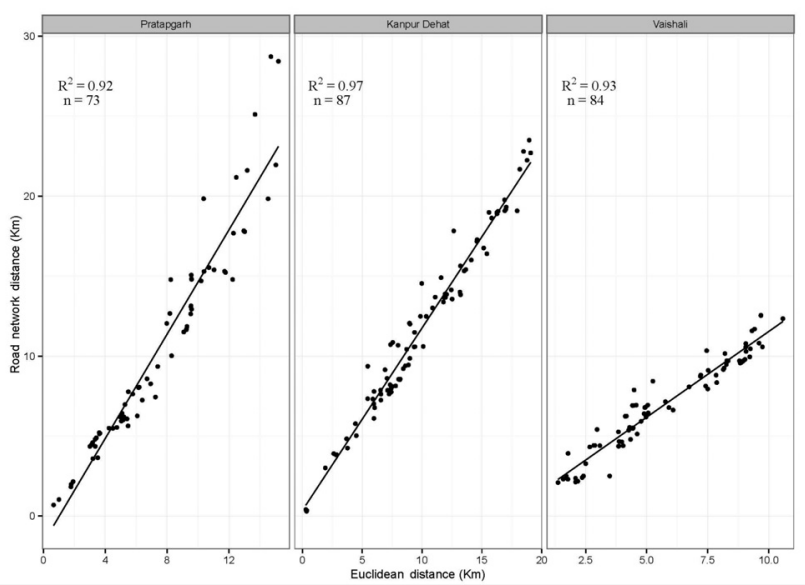

Fig. 2. Scatter plot and "best fit" line between the road network distance and the Euclidean distance for the three study sites.

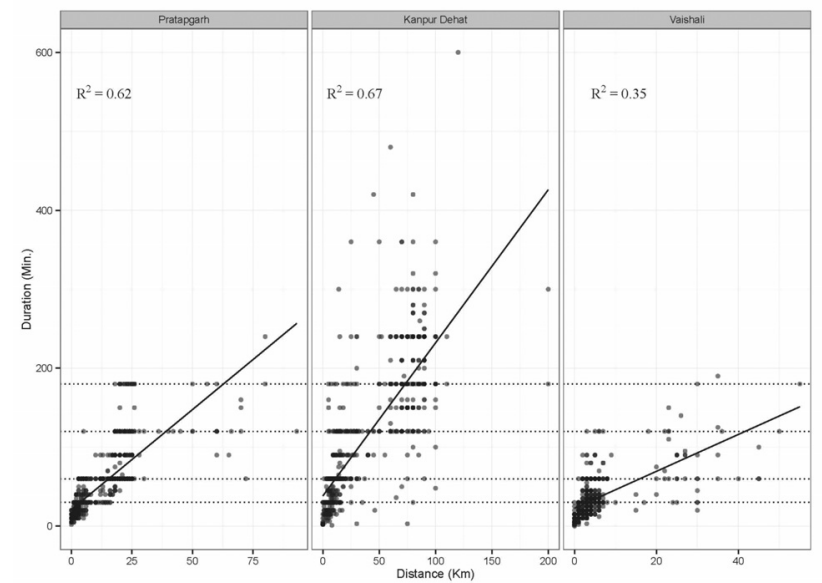

Fig. 3. Scatter plot and "best fit" line between distance travel and time taken in min. The dotted horizontal lines indicate "round offs" to the nearest 30, 60, 120 and $180 \mathrm{~min}$. Transparency (reflected by the number of dots in the same place) was used to stress that most of the observations were hour round-offs. It should also be noted that the dots on the dotted lines are mostly completely opaque (black), indicate at least three points overlap there.

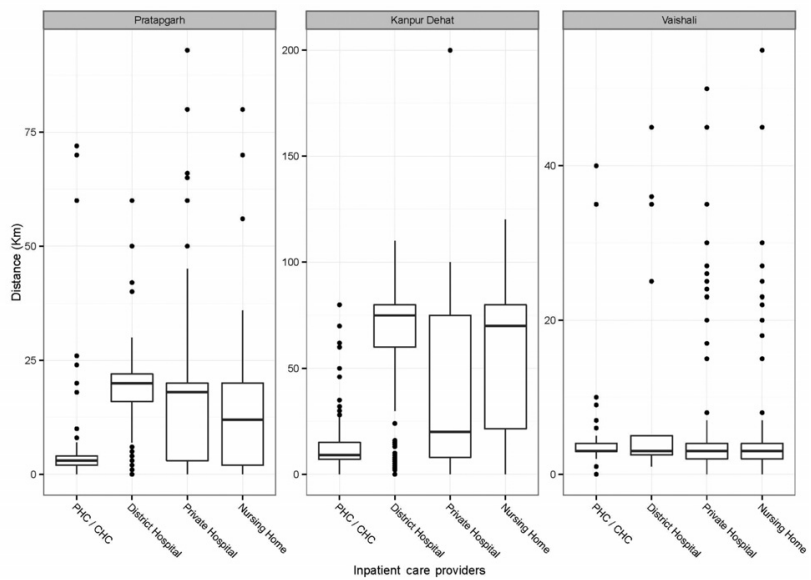

Fig. 4. Boxplots representing travelling distance to seek care from different in-patient care providers.
Travel distance to in-patient care facilities

As evident from Fig. 4, there are huge disparities between the study sites and thus difficult to generalise proximity with respect to in-patient care. People from Pratapgarh and Kanpur Dehat must travel more than people from Vaishali to seek in-patient care. However, as distances would be too large (approximately 100 $\mathrm{km}$ ) if those from Fig. 4 were used, this study assumed that $10 \mathrm{~km}$ would be appropriate as catchment area because this would result in a two-fold increment in catchment area (i.e. $5 \mathrm{~km}$ ) of dominant out-patient providers (for non-qualified doctors, who are the dominant out-patient care providers). As evident from Fig. 4, there is serious lack of nearby in-patient facilities in the Kanpur Dehat site. On the other hand, inpatient facilities were found to be more closely situated in Vaishali than in any of the other two sites.

Potential spatial access using 3SFCA method

PHCs were excluded from analysis, because in most of the cases they were found to have no functional inpatient care facilities. Since all the in-patient facilities were concentrated in the market areas, spatial access in all three sites were concentric, i.e. gradually decreasing outwards. Higher values of SPAR and SPAI indicate better spatial access. Under Indian Governmental norms, there should be one CHC for 120,000 people. Therefore, this study calculated shortage of in-patient care according to this reference. Following this criterion, Vaishali site did not show any shortage of inpatient care. As evident from Figs. 5 and 6, the distribution of SPAR and SPAI is not uniform. Since SPAR is the normalised SPAI values, variations in SPAR values are attributed to the distribution shapes of SPAI values. In the case of Kanpur Dehat, the SPAR was found to be quite high and correspond to the global mean of SPAI, which lies near the lowest values causing high SPAR values. This is an important observation, since it indicates that SPAR is not comparable and can only be interpreted exclusively for one study area at a time and therefore has little value for comparison purposes. In Kanpur Dehat, 90\% of the villages showed shortage of in-patient facilities. Similarly, in Pratapgarh, approximately $40 \%$ of the villages had in-patient facility shortage.

This research identifies that it is important to assess the explanatory variable, which affects the variation in spatial access. As expected, the spatial autocorrelation for distance to nearest UA was found to be very high and not significant, or only marginally significant, as 


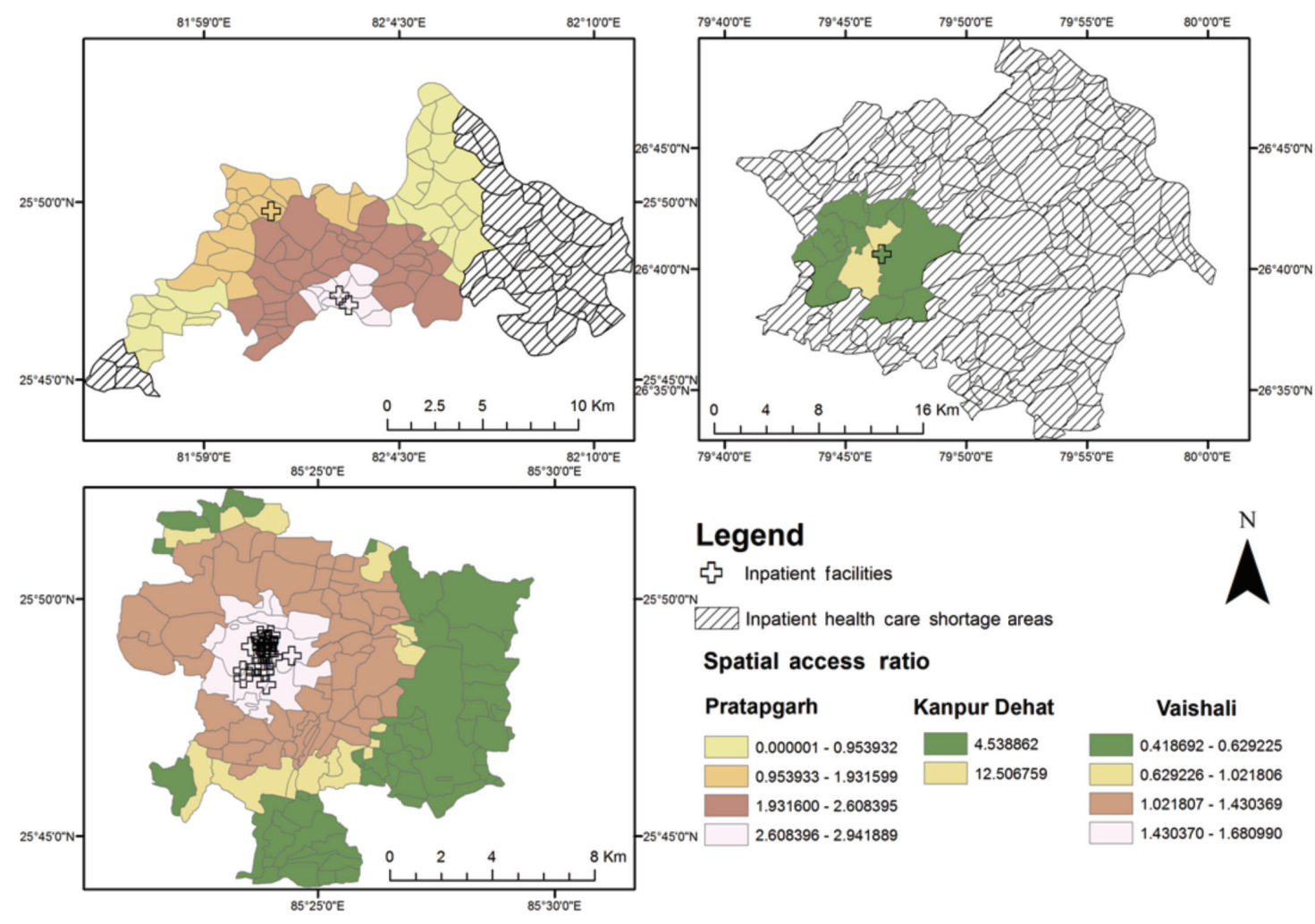

Fig. 5. Spatial access ratios and in-patient health care shortage areas for Pratapgarh, Kanpur Dehat and Vaishali.

seen in Kanpur Dehat and Pratapgarh (for other socioeconomic variables see appendix, Table 1). The positive spatial autocorrelation in Kanpur Dehat (except for N_DIST) was due to two market areas contiguous to each other, i.e. Rasoolabad and Asalatganj, but by deleting the latter, the spatial autocorrelation was removed from all the parameters (see Appendix, Table 1). Therefore, Kruskal-Wallis test was run for Kanpur Dehat after deleting Asalatganj. For the nearest distance to UAs, only randomly selected $10 \%$ of the total observations were used $(n=12)$ and the number of observations were kept same as the site with a minimum number of observations to make it a balanced design. Although this might not remove spatial autocorrelation completely (Dale and Fortin, 2002), the variation was considered true since the observations varied greatly among the study sites (Fig. 6d). The Kruskal-Wallis test showed that at least one of the sites was different from the other with respect to all the parameters studied (Appendix, Table 2). Although, their deviation from each other did not show the same trend as was found with spatial access except for the nearest distance (Appendix, Table 2 and Fig. 6d). The nearest distance from UA was the highest (mean $=55$ $\mathrm{km}$ ) for Kanpur Dehat followed by Pratapgarh (mean $=42 \mathrm{~km})$ and Vaishali $($ mean $=34 \mathrm{~km})$; this relation is exactly opposite to what was found with spatial access in the three study sites. However, although this analysis does not give a direct relation between spatial access values; it does give enough evidence to say that spatial access co-vary with distance to the UA.

The reason of this negative relation can be attributed to easier access to medicine, doctors, medical instruments, which are all concentrated in major UAs. Since the private medical facilities are profit-oriented they are centred in the market areas, which indicate that the further situated a village is from the urban area, the lower its access to medical facilities. Furthermore, none of the socio-economic variables studied here found to have effect on spatial access.

\section{Discussion}

Spatial access is an important part of accessing health care in low- and middle-income countries (Peters et al., 2008) and measuring accessibility to health care facilities contributes to a wider understanding of the performance of health care systems, which facilitates the development of evidence-based health policies (Black et al., 2004). There are few studies which considered multiple sites with mixed approaches like the one used in this paper to assess 

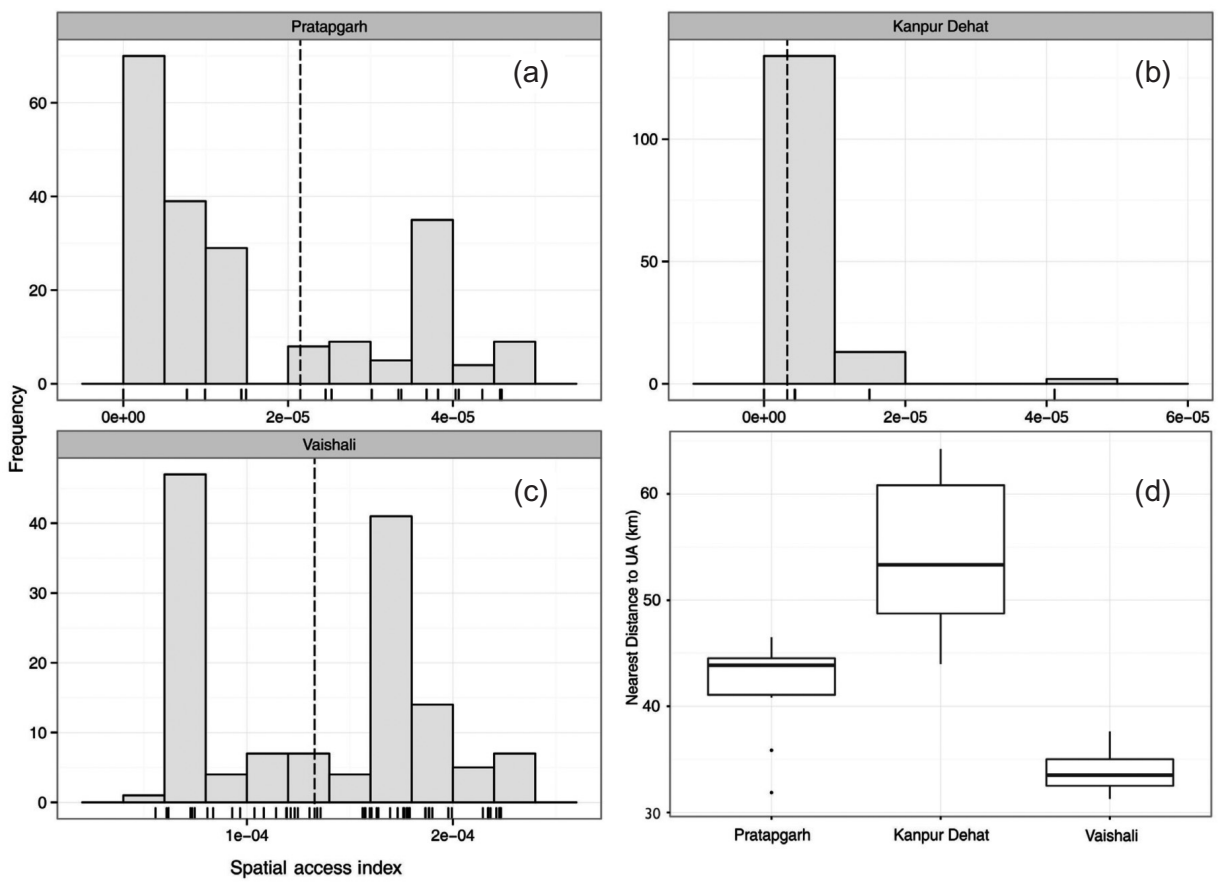

Fig. 6. Spatial access ratios and in-patient health care shortage areas for Pratapgarh, Kanpur Dehat and Vaishali. The site-wise distribution of SPAI values: Pratapgarh $(\mathrm{n}=124)(\mathrm{a})$, Kanpur Dehat $(\mathrm{n}=140)(\mathrm{b})$ and Vaishali $(\mathrm{n}=128)(\mathrm{c})$ with the vertical dotted lines representing the mean values. Fig. $6 \mathrm{~d}$ shows the nearest distance $(\mathrm{km})$ to an urban agglomeration for 36 randomly selected observations (12 from each site).

spatial access. Comparison of three different sites actually helped in understanding various spatial issues related to access to in-patient health care. The methodology for assessing spatial access is frequently changing and the floating catchment methods, although previously introduced for job accessibility analysis (Peng, 1997), are relatively new in public health (Luo, 2004). The 3SFCA method constitutes good progress, but we feel that these methodologies will keep changing before a stable approach can be found. The reason of this might be due to definition of "access"; which is also still evolving (Aday and Andersen, 1974; Penchansky and Thomas, 1981; Guagliardo, 2004; McIntyre et al., 2009; Levesque et al., 2013). Methodological progress depends on newly added technology as well as dissemination of previous publications from different parts of the world. Most new methodologies are developed and tested in developed countries, while the significantly different scenarios of the third world are often ignored. The need to determine spatial access is undeniable in every part of the world, so suitable modifications according to the area studied are always necessary. Furthermore, there are no off-the-shelf tools that can facilitate the implementation of these methodologies, partly because of the continuing pace of methodological changes. One solution is to adapt already available tools for new uses. This might include application of open-source tools such as R (R Development Core Team, 2012) used in this study. The power of $\mathrm{R}$ lies in the ease of handling non-spatial as well as spatial data making it unnecessary to switch platforms between analyses.

Spatial access in rural and poor India is still very far from satisfactory. Distance to UA, which is a major

Table 2. Kruskal-Wallis statistics for the study parameters.

\begin{tabular}{lccc}
\hline Parameter & $\chi^{2}$ & Degree of freedom & P-value \\
\hline TOT_POP $^{a}$ & 16.07 & 2 & $<0.001$ \\
TOT_LIT $^{b}$ & 23.80 & 2 & $<0.001$ \\
TOT_ILLT $^{c}$ & 20.14 & 2 & $<0.001$ \\
TOT_NNW $^{d}$ & 20.17 & 2 & $<0.001$ \\
TOT_CULT $^{e}$ & 68.64 & 2 & $<0.001$ \\
N_DIST $^{\natural}$ & 27.44 & 2 & $<0.001$
\end{tabular}

${ }^{\mathrm{a}}$ Total population; ${ }^{\mathrm{b}}$ total literacy; 'total illiteracy; ${ }^{\mathrm{d}}$ total non-workers; ${ }^{\mathrm{e}}$ total cultivators; ${ }^{\mathrm{f}}$ distance to nearest urban agglomeration (UA). 
constraint to access, had also been identified by Krishna and Ananthpur (2013). In-patient facilities are concentrated in urban or semi-urban areas to maximise profit leading to extra transportation costs for already poor people, while people living in urban areas, who are often better off, have better accessibility and less transportation costs. Furthermore, such scenarios push poor people to even poorer conditions due to overburdening health care expenses. As shown in this study, and in other countries (Chan et al., 2006; Onega et al., 2008; Wang et al., 2008; Luo and Qi, 2009; Wan et al., 2012a) as well as in India (Baru et al., 2010; Krishna and Ananthpur, 2013), the less developed the area, the lesser the spatial accessibility. Disparities between Uttar Pradesh and Bihar were also reflected by Balarajan et al. (2011), who have reported higher mortality per 1,000 live-births in Uttar Pradesh compared to Bihar. The solution, however, is not forcing allocations of private health care facilities but to allocate public in-patient care appropriately and making sure they are properly staffed.

Travel distance to health care providers is an important parameter as it reveals how much people have to travel when seeking care. In general, private practices are farther located than Government facilities, so people have to travel more when seeking care at private hospitals than at Government ones. This can be attributed to the fact that private providers are profit-oriented; therefore, they are always located around the UAs and thus farther from the rural areas. The district hospitals are closer to the private providers, which indicate that the latter are always situated in UAs.

An oversized catchment area would show results indicating that none of the villages are deprived of appropriate spatial access. Under this presumption, they would all appear to be able to utilise a hospital, even if it were situated $100 \mathrm{~km}$ away. Thus, a high value for the catchment area could give the false impression of excellent spatial access. Since there is no concensus of what should be the catchment area of inpatient health care facilities, we chose the practical estimate of $10 \mathrm{~km}$ as catchment area of in-patient health care facilities for this study.

The primary health care of rural India depends on unqualified local medical practitioners (LMPs). As revealed by Das et al. (2012), 70\% of the primary care visits in rural India are handled by these LMPs. In this scenario, inclusion of LMPs as primary care providers would give an inappropriate indication of the health care situation. On the other hand, if the LMPs were excluded, there might be no primary care providers at all (as in the Kanpur Dehat site) except in Government
CHCs. Access to in-patient care was studied bearing this in mind. However, if access to out-patient care would be studied, inclusion of such informal health care providers (LMPs) should be made.

The limitation of this study, as with any other studies with defined boundaries, is that there might be inpatient facilities just outside the boundary, which could not be included even if a strong flow of patients would choose this facility as it might be closer. However, it is also important to define boundaries, without which it is not possible to carry out any spatial access study. Furthermore, since household survey was a RCT design and covered only members affiliated to self-help groups, their results can not be generalised to the whole population at the three sites. However, given the paucity of more representative data, the household survey information provided very useful insights (Panda et al., 2013).

\section{Conclusions}

This study primarily points out on the geographical inequities in accessing in-patient care in rural, northern India. The overall scenario indicates poor access to in-patient care. The inequities were realised for intrasite as well as inter-site scenarios. Conspicuous, concentric patterns indicate clustering of all the facilities to mainly one market area. This study explored covariability of socio-economic variables to spatial access, which indicates that spatial access primarily depends on distance to nearest urban agglomerations. Additionally, we found that allocation of in-patient facilities has less to do with population needs than maximization of provider profits. Independence of allocation of care facilities from population needs is reflected by observed absence of impact by socio-economic conditions on spatial access. The findings can be summarised as:

(i) serious spatial access disparities were found between two poorest states of India;

(ii) distance to nearest urban centre is negatively associated with spatial access; and

(iii) equal spatial access to care requires needs-adjusted allocation of health care facilities.

\section{Acknowledgements}

Research reported in this article has been funded by the European Union's FP7 programme, project-Community-Based Health Insurance in India, HEALTH-F2-2009-223518. We are grateful to the two anonymous reviewers for their very useful comments. 


\section{References}

Aczel A, Sounderpandian V, 2006. Complete business statistics, Tata-McGraw Hill Publication, 918 pp.

Aday LA, Andersen R, 1974. A framework for the study of access to medical care. Health Serv Res 9, 208-220.

Balarajan Y, Selvaraj S, Subramanian SV, 2011. Health care and equity in India. Lancet 377, 505-515.

Banerjee A, Deaton A, Duflo E, 2004. Health, health care, and economic development. Am Econ Rev 94, 326-330.

Baru R, Acharya A, Acharya S, Shiva Kumar AK, Nagaraj K, 2010. Inequities in access to health services in India: caste, class and region. Econ Polit Wkly 45, 49-58

Bivand R, 2013. Spatial dependence: weighting schemes, statistics and models. R package version 0.5-68.

Bivand R, Rundel C, 2013. Rgeos: interface to geometry engine - Open source (GEOS). R package version 0.3-2.

Black M, Ebener S, Aguilar Najera P, Vidaurre M, El Morjani Z, 2004. Using GIS to measure physical accessibility to health care. presented at the international health users conference, Washington DC, p. 22.

Chan L, Hart LG, Goodman DC, 2006. Geographic access to health care for rural medicare beneficiaries. J Rural Health 22, 140-146.

Census of India, 2011. Provisional population totals, urban agglomerations and cities, govt of India. Available at: http://censusindia.gov.in/2011-prov-results/paper2/data_files/ India2/1.\%20Data\%20Highlight.pdf (accessed on March 2013).

Dale MRT, Fortin M-J, 2002. Spatial autocorrelation and statistical tests in ecology. Ecoscience 9, 162-167.

Das J, Holla A, Das V, Mohanan M, Tabak D, Chan B, 2012. In urban and rural india, a standardized patient study showed low levels of provider training and huge quality gaps. Health Aff (Millwood) 31, 2774-2784.

De Costa A, Diwan V, 2007. "Where is the public health sector?" Public and private sector healthcare provision in Madhya Pradesh, India. Health Policy 84, 269-276.

Deshpande K, RaviShankar, Diwan V, Lönnroth K, Mahadik VK, Chandorkar RK, 2004. Spatial pattern of private health care provision in Ujjain, India: a provider survey processed and analysed with a geographical information system. Health Policy 68, 211-222.

Diez D, Barr C, Çetinkaya-Rundel M, 2013. OpenIntro statistics: second edition. CreateSpace independent publishing platform, 426 pp.

Dixit S, Panda P, 2013. Spatial research methodology supplementing cluster randomized control trials: learning from a study of community-based health insurance schemes in India. Int J Geoinformatics 9, 31-38.

Doyle C, Panda P, de Poel EV, Radermacher R, Dror DM, 2011. Reconciling research and implementation in micro health insurance experiments in India: study protocol for a randomized controlled trial. Trials 12, 224.

Guagliardo MF, 2004. Spatial accessibility of primary care: concepts, methods and challenges. Int J Health Geogr 3, 3.

Higgs G, 2004. A literature review of the use of GIS-based measures of access to health care services. Health Serv Outcomes Res Methodol 5, 119-139.

Horton R, Das P, 2011. Indian health: the path from crisis to progress. Lancet 377, 181-183.

Joseph AE, Phillips DR, 1984. Accessibility and utilization: geographical perspectives on health care delivery. SAGE, 214 pp. Krishna A, Ananthpur K, 2013. Globalization, distance and disease: spatial health disparities in rural India. Millenn Asia 4, 3-25.

Kwan M-P, 1998. Space time and integral measures of individual accessibility: a comparative analysis using a point based framework. Geogr Anal 30, 191-216.

Levesque JF, Harris MF, Russell G, 2013. Patient-centred access to health care: conceptualising access at the interface of health systems and populations. Int J Equity Health 12, 18.

Luo W, 2004. Using a GIS-based floating catchment method to assess areas with shortage of physicians. Health Place 10, 1-11. Luo W, Qi Y, 2009. An enhanced two-step floating catchment area (E2SFCA) method for measuring spatial accessibility to primary care physicians. Health Place 15, 1100-1107.

McGrail MR, Humphreys JS, 2009. Measuring spatial accessibility to primary care in rural areas: improving the effectiveness of the two-step floating catchment area method. Appl Geogr 29, 533-541.

McIntyre D, Thiede M, Birch S, 2009. Access as a policy-relevant concept in low- and middle-income countries. Health Econ Policy Law 4, 179-193.

McLafferty SL, 2003. Gis and health care. Annu Rev Public Health 24, 25-42.

Ministry of Health and Family Welfare, 2012. Rural health care system in India.

Onega T, Duell EJ, Shi X, Wang D, Demidenko E, Goodman D, 2008. Geographic access to cancer care in the US. Cancer 112, 909-918.

Panda P, Chakraborty A, Dror DM, Bedi AS, 2013. Enrolment in community-based health insurance schemes in rural Bihar and Uttar Pradesh, India. Health Policy Plan 26, 1-15.

Peel MC, Finlayson BL, McMahon TA, 2007. Updated world map of the Köppen-Geiger climate classification. Hydrol Earth Syst Sci 11, 1633-1644.

Penchansky R, Thomas JW, 1981. The concept of access: definition and relationship to consumer satisfaction. Med Care 19, 127-140.

Peng Z-R, 1997. The jobs-housing balance and urban commuting. Urban Studies 34, 1215-1235.

Peters DH, Garg A, Bloom G, Walker DG, Brieger WR, Hafizur Rahman M, 2008. Poverty and access to health care in devel- 
oping countries. Ann N Y Acad Sci 1136, 161-171.

Qadeer I, 2011. The challenge of building rural health services. Indian J Med Res 134, 591-593.

QGIS Development Team, 2013. QGIS geographic information system. Open Source Geospatial Foundation.

R Development Core Team, 2012. R: a language and environment for statistical computing. R Foundation for Statistical Computing. Vienna: R Foundation for Statistical Computing.

Radke J, Mu L, 2000. Spatial decompositions, modeling and mapping service regions to predict access to social programs. Int J Geogr Inf Sci 6, 105-112.

Sengupta A, Prasad V, 2011. Towards a truly universal Indian health system. Lancet 377, 702-703.

Silverman BW, 1986. Density estimation for statistics and data analysis. Chapman and Hall/CRC, New York.

Spencer J, Angeles G, 2007. Kernel density estimation as a technique for assessing availability of health services in Nicaragua. Health Serv Outcomes Res Methodol 7, 145-157.

Wan N, Zou B, Sternberg T, 2012a. A three-step floating catchment area method for analyzing spatial access to health serv- ices. Int J Geogr Inf Sci 26, 1073-1089.

Wan N, Zhan FB, Zou B, Chow E, 2012b. A relative spatial access assessment approach for analyzing potential spatial access to colorectal cancer services in Texas. Appl Geogr 32, 291-299.

Wang F, 2002. Where the jobs are: employment access and crime patterns in Cleveland. Ann Assoc Am Geogr 92, 435.

Wang F, Luo W, 2005. Assessing spatial and nonspatial factors for healthcare access: towards an integrated approach to defining health professional shortage areas. Health Place 11, 131-146.

Wang F, McLafferty S, Escamilla V, Luo L, 2008. Late-stage breast cancer diagnosis and health care access in Illinois. Prof Geogr 60, 54-69.

Yang D-H, Goerge R, Mullner R, 2006. Comparing GIS-based methods of measuring spatial accessibility to health services. J Med Syst 30, 23-32.

Yao J, Murray AT, Agadjanian V, 2013. A geographical perspective on access to sexual and reproductive health care for women in rural Africa. Soc Sci Med 96, 60-68. 


\section{Appendix}

Table 1. Moran's I for spatial autocorrelation and P-values for all three sites.

1a. Pratapgarh

\begin{tabular}{lcc}
\hline Parameter & Moran's $I$ & P-value \\
\hline TOT_POP $^{\mathrm{a}}$ & 0.01 & 0.36 \\
TOT_LIT $^{\mathrm{b}}$ & 0.00 & 0.44 \\
TOT_ILLT $^{\mathrm{c}}$ & 0.02 & 0.32 \\
TOT_NNW $^{\mathrm{d}}$ & 0.00 & 0.39 \\
TOT_CULT $^{\mathrm{c}}$ & 0.12 & 0.01 \\
N_DIST $^{f}$ & 0.96 & 0.00 \\
\hline
\end{tabular}

1.2b. Kanpur Dehat*

\begin{tabular}{lcc}
\hline Parameter & Moran's $I$ & P-value \\
\hline TOT_POP $^{a}$ & 0.04 & 0.14 \\
TOT_LIT $^{b}$ & 0.04 & 0.14 \\
TOT_ILLT $^{c}$ & 0.04 & 0.13 \\
TOT_NNW $^{d}$ & 0.05 & 0.10 \\
TOT_CULT $^{c}$ & 0.00 & 0.46 \\
N_DIST $^{\mathrm{f}}$ & 0.96 & 0.00
\end{tabular}

1.1b. Kanpur Dehat

\begin{tabular}{lcc}
\hline Parameter & Moran's $I$ & P-value \\
\hline TOT_POP $^{\mathrm{a}}$ & 0.06 & 0.05 \\
TOT_LIT $^{\mathrm{b}}$ & 0.06 & 0.05 \\
TOT_ILLT $^{c}$ & 0.07 & 0.04 \\
TOT_NNW $^{\mathrm{d}}$ & 0.07 & 0.03 \\
TOT_CULT $^{\mathrm{c}}$ & 0.00 & 0.45 \\
N_DIST $^{\mathrm{f}}$ & 0.96 & 0.00
\end{tabular}

1c. Vaishali

\begin{tabular}{lcc}
\hline Parameter & Moran's $I$ & P-value \\
\hline TOT_POP $^{\mathrm{a}}$ & -0.04 & 0.71 \\
TOT_LIT $^{\mathrm{b}}$ & -0.03 & 0.67 \\
TOT_ILLT $^{\mathrm{c}}$ & -0.03 & 0.70 \\
TOT_NNW $^{\mathrm{d}}$ & -0.02 & 0.63 \\
TOT_CULT $^{\mathrm{c}}$ & -0.03 & 0.68 \\
N_DIST $^{\mathrm{f}}$ & -0.02 & 0.63 \\
\hline
\end{tabular}

Total population; ${ }^{\mathrm{b}}$ total literacy; ${ }^{\mathrm{c}}$ total illiteracy; ${ }^{\mathrm{d}}$ total non-workers; ${ }^{\mathrm{e}}$ total cultivators; ${ }^{\mathrm{f}}$ distance to nearest urban agglomeration (UA). *After deletion of Asalatganj (with spatial autocorrelation removed after deletion except for nearest UA).

Table 2. Pairwise-Wilcoxon rank-sum comparisons (P-values).

\begin{tabular}{llcc}
\hline Parameter & Site & Moran's $I$ & P-value \\
\hline TOT_POP & Pratapgarh & $<0.001$ & - \\
& Vaishali & 1 & 0.006 \\
TOT_LIT & Pratapgarh & $<0.001$ & - \\
& Vaishali & 0.005 & 0.64 \\
TOT_ILLT & Pratapgarh & 0.031 & - \\
& Vaishali & 0.07 & $<0.001$ \\
& Pratapgarh & $<0.001$ & - \\
TOT_NNW & Vaishali & 1 & 0.001 \\
& Pratapgarh & $<0.001$ & - \\
TOT_CULT & Vaishali & $<0.001$ & 0.31 \\
& Pratapgarh & $<0.001$ & - \\
N_DIST & Vaishali & $<0.001$ & 0.002 \\
\hline
\end{tabular}

